J. Lake Sci.(湖泊科学), 2019, 31(1): 113-123

DOI 10. 18307/2019. 0111

(c) 2019 by Journal of Lake Sciences

\title{
珠江三角洲湖库水体嗅味物质分布及其去除
}

\author{
刘祖发 ${ }^{1,2}$, 陈记臣 ${ }^{1,2}$, 卓文珊 $3^{* *}$, 曾 珂 ${ }^{1,2}$, 梁廖逢 ${ }^{1,2}$, 刘 茹 ${ }^{1,2}$ \\ (1: 中山大学水资源与环境研究中心,广州 510275 ) \\ (2:华南地区水循环与水安全广东省普通高校重点实验室,广州 510275$)$ \\ (3:中山大学测试中心,广州 510275)
}

\begin{abstract}
摘 要: 采用固相微萃取一气质联用技术对珠江三角洲 9 个城市共 48 个湖库水体中的土臭素 (GSM)、2-甲基异茨醇 (MIB) 和 2,3,6-三氯苯甲醚 (TCA) 等 5 种嗅味物质进行检测, 并探讨污水处理厂和自来水厂处理工艺中嗅味物质的浓度 变化规律, 同时研究强化混凝沉淀工艺对嗅味物质的去除效果. 结果表明: 珠江三角洲湖库水体中嗅味问题最严重的是 广州、佛山、肇庆、东莞、惠州和深圳 6 个城市, 各市湖库的嗅味物质平均浓度为 $70.93 \sim 116.61 \mathrm{ng} / \mathrm{L}$; 中山、珠海与江门 3 个城市的嗅味问题不明显, 各市湖库的嗅味物质平均浓度为 $22.78 \sim 58.82 \mathrm{ng} / \mathrm{L}$; 珠江三角洲的湖库中浓度最高的嗅味物 质是 MIB、TCA 和 GSM,这 3 种唤味物质占了嗅味物质总浓度的 $80.18 \% \sim 100.00 \%$; 污水处理厂和自来水厂中的处理工艺 对嗅味物质均有一定的去除效果, 嗅味物质的去除主要发生在混凝沉淀阶段; 污水处理厂对 MIB、TCA 和 GSM 的平均去 除率分别为 $53.55 \% 、 57.40 \%$ 和 $72.90 \%$; 自来水厂对 MIB、TCA 和 GSM 的平均去除率分别为 $64.14 \% 、 69.63 \%$ 和 $36.86 \%$; 强 化混凝沉淀实验中, 当嗅味物质初始浓度为 $200 \mathrm{ng} / \mathrm{L}$ 时, $\mathrm{Al}_{2} \mathrm{O}_{3}$ 投加量为 $13.75 \mathrm{mg} / \mathrm{L}$ 可使得混凝沉淀工艺对嗅味物质的 去除效果最佳且保证铝盐不超标, 嗅味物质浓度增大时 $\mathrm{Al}_{2} \mathrm{O}_{3}$ 投加量也需增大; 反应体系的 $\mathrm{pH}$ 值处于 $5 \sim 8$ 时, 混凝沉淀 工艺对嗅味物质的去除效果最佳;混凝摚拌速率越快, 嗅味物质的去除率越高且越快达到稳定.
\end{abstract}

关键词: 珠江三角洲; 湖库; 强化混凝沉淀; 嗅味物质; 固相微萃取一气质联用

\section{Distribution of odor and taste compounds in the lakes and reservoirs of Pearl River Delta and the removal efficiency in source discharges}

\author{
LIU Zufa ${ }^{1,2}$, CHEN Jichen ${ }^{1,2}$, ZHUO Wenshan ${ }^{3 * *}$, ZENG Ke ${ }^{1,2}$, LIANG Liaofeng ${ }^{1,2} \&$ LIU Ru ${ }^{1,2}$ \\ (1: Center for Water Resource and Environment, Sun Yat-sen University, Guangzhou 510275, P.R. China) \\ (2: Key Laboratory of Water Cycle and Water Security in Southern China of Guangdong Higher Education Institutes, Guang- \\ zhou 510275, P.R.China) \\ (3: Instrumental Analysis \& Research Center, Sun Yat-sen University, Guangzhou 510275, P.R. China)
}

Abstract: With the continuous improvement of people's living standards, residents put forward higher requirements for water quality, and pay more attention to odor and taste problem. Concentrations of odor and taste compounds in 48 lakes and reservoirs of 9 cities in Pearl River Delta were detected with the method of Solid Phase MicroExtraction-Gas Chromatography/Mass Spectrometry (SPME-GC/MS) technique. The changes of odor and taste compounds concentrations along the water treatment processes of wastewater treatment plants and water supply plants, as well as the removal effect of odor and taste compounds by enhanced coagulation-sedimentation process were studied. The results showed that: the most serious stench problem was the lakes and reservoirs of Guangzhou, Foshan, Zhaoqing, Dongguan, Huizhou and Shenzhen. The average concentrations of odor and taste compounds in the lakes and reservoirs of these 6 cities were from 70.93 to $116.61 \mathrm{ng} / \mathrm{L}$. And the stench problem was not obvious in the lakes and reservoirs of Zhongshan, Zhuhai and Jiangmen. The average concentrations of odor and taste compounds in the lakes and reservoirs of these 3 cities were from 22.78 to $58.82 \mathrm{ng} / \mathrm{L}$. 2-methylisoborneol ( MIB ) , 2, 3, 6-trichloroanisole (TCA) and Geosmin ( GSM) were the main odor and taste compounds in all lakes and reservoirs, which accounted for $80.18 \%-100.00 \%$ of total odor and taste

* 国家自然科学基金项目 (41301627)资助. 2018-04-03 收稿;2018-05-28 收修改稿. 刘祖发 (1961 ), 男,博士, 副 教授;E-mail: eeslzf@ mail.sysu.edu.cn.

** 通信作者; E-mail:zadeozws@ mail.sysu.edu.cn. 
compounds. The water treatment progresses of wastewater treatment plants and water supply plants could considerably remove odor and taste compounds. Coagulation-sedimentation process had the greatest contribution to the removal of odor and taste compounds. In the wastewater treatment plants, the average removal rates of MIB, TCA and GSM were 53.55\%, 57.40\% and 72.90\%, respectively. In the water supply plants, the average removal rates of MIB, TCA and GSM were 64.14\%, 69.63\% and 36.86\%, respectively. In the enhanced coagulation-sedimentation experiment, for the initial concentrations of odor and taste compounds of $200 \mathrm{ng} /$ $\mathrm{L}$, coagulation-sedimentation process could reach the greatest removal effect of them, and aluminum was not excessive as well when the coagulant $\left(\mathrm{Al}_{2} \mathrm{O}_{3}\right)$ dosage was $13.75 \mathrm{mg} / \mathrm{L}$. When the concentrations of odor and taste compounds increase, the dosage of $\mathrm{Al}_{2} \mathrm{O}_{3}$ should increase, too. When the $\mathrm{pH}$ value of the reaction system was between 5 and 8 , coagulation-sedimentation process could reach the greatest removal effect of odor and taste compounds. The faster the stirring rate of coagulation was, the higher the removal rate of odor and taste compounds reached, and the faster the stability reached.

Keywords: Pearl River Delta; lakes and reservoirs; enhanced coagulation-sedimentation; odor and taste compounds; SPMEGC/MS

水体嗅味问题普遍存在于我国的湖泊水库等水体中 ${ }^{[1]}$. 近年来, 我国江苏太湖 ${ }^{[2]}$ 、云南滇池 ${ }^{[3]}$ 和湖北熊 河水库 ${ }^{[4]}$ 等湖库均有水体异嗅事件发生. 引起水体嗅味的物质主要为土臭素 (Geosmin, GSM)、2-甲基异茨 醇 (2-methylisoborneol，MIB)、2-异丙基-3-甲氧基吡嗪 (2-isopropyl-3-methoxypyrazine，IPMP)、2-异丁基-3-甲 氧基吡嗪 (2-isobutyl-3-methoxypyrazine, IBMP) 以及 2,3,6-三氯苯甲醚 $\left(2,3,6\right.$-trichloroanisole, TCA) 等 ${ }^{[5]}$. 嗅 味物质不仅会使水体产生异嗅味从而给人带来感官的不适 ${ }^{[6]}$, 而且对生物具有较高的毒性 ${ }^{[7]}$. 污水处理厂 和自来水厂的混凝沉淀等处理工艺对嗅味物质的浓度变化有一定的影响 ${ }^{[8]}$.

由于异嗅味是湖库等水体的常见问题, 国内外已有不少对嗅味物质分布与去除的研究. Zuo 等 ${ }^{[4]}$ 研究了 湖北熊河水库中的嗅味物质, 发现 GSM 和 MIB 等嗅味物质主要由蓝藻和细菌等产生; Tung 等 ${ }^{[9]}$ 和 Chiu 等 ${ }^{[10]}$ 研究发现台湾水库群中已普遍存在着嗅味物质,且自来水厂的沉淀工艺能够去除约 $50 \%$ 的嗅味物质; Westerhoff 等 ${ }^{[11]}$ 调查了美国亚利桑那州 3 个水库中的嗅味物质, 发现嗅味物质在春、夏季浓度较高而秋、冬 季浓度较低, 且与细菌及藻类的生长繁殖有着密切关系. 在国内, 目前常见的是对单个湖库中嗅味物质进行 研究, 但对区域的嗅味物质分布研究尚不多见. 梁恒等 ${ }^{[12]}$ 和 Hageskal 等 $^{[13]}$ 通过对不同水处理工艺流程的研 究, 发现常规混凝沉淀工艺对藻类的去除率小于 $60 \%$; 刘成等 ${ }^{[14]}$ 分析了在藻类暴发时, 常规混凝沉淀工艺在 投药量很大的情况下能去除约 $80 \%$ 的藻类. 国内学者对嗅味物质的研究多为混凝沉淀工艺对藻类的去除研 究,鲜有对污水处理厂和自来水厂工艺流程以及强化混凝沉淀工艺对嗅味物质去除的相关探讨.

珠江三角洲是全国经济发展最迅速的地区之一 ${ }^{[15]}$. “珠江三角洲经济区” 的范围主要为珠江沿岸的广 州、深圳、佛山、珠海、东莞、中山、江门、惠州和肇庆 9 个城市. 为探讨珠江三角洲湖库中 GSM、MIB、IPMP、 IBMP 和 TCA 这 5 种嗅味物质的分布情况, 以及污水处理厂和自来水厂各级处理工艺中嗅味物质的浓度变 化规律, 并研究强化混凝沉淀工艺对嗅味物质的去除效果, 本文在珠江三角洲的 9 个城市中选取了具有代 表性的 48 个湖库进行水体样品采集, 分析了珠江三角洲的污水处理厂 $\mathrm{A}$ 和 $\mathrm{B} 、$ 自来水厂 $\mathrm{C}$ 和 $\mathrm{D}$ 的处理工艺 对嗅味物质的去除率, 同时着重于研究混凝剂投加量、嗅味物质初始浓度、反应体系 $\mathrm{pH}$ 值以及摚拌速率对 强化混凝沉淀工艺除嗅的影响, 以找到混凝沉淀除嗅效果最佳的控制条件, 为有效去除水中的异嗅味提供 依据.

\section{1 材料与方法}

\section{1 样品采集}

本文针对珠江三角洲的湖库水体、污水处理厂与自来水厂水体进行研究, 对 9 个城市中的 48 个湖库、2 个污水处理厂与 2 个自来水厂进行水体样品采集 (图 1), 表 1 为本研究的湖库采样点编号对应信息. 于 2017 年 12 月, 将有机玻璃采水器置于水面以下 $0.5 \mathrm{~m}$ 处采集水样, 先用水样润洗采样器和采样瓶 3 遍, 再将 水样装于 $100 \mathrm{ml}$ 棕色玻璃样品瓶中; 对于污水处理厂和自来水厂的进水和出水等在水龙头采集的水样,先 打开水龙头使水样缓慢流出约 $5 \mathrm{~min}$ 后再采集. 样品瓶中装满水, 不留空气, 采样后尽快将样品置于 $4^{\circ} \mathrm{C}$ 的 冰箱中保存, 待测定时再恢复至室温. 


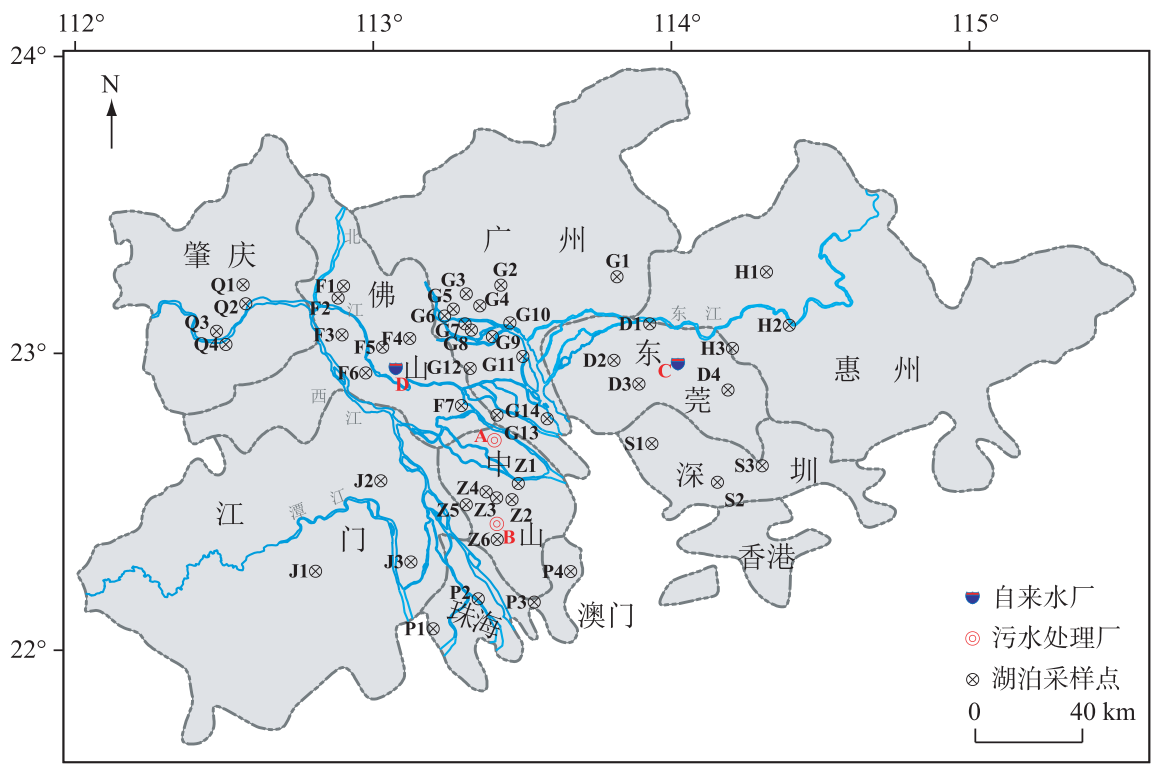

图 1 珠江三角洲湖库采样点、污水处理厂与自来水厂布设

Fig. 1 Location of sampling sites of the lakes and reservoirs, wastewater treatment plants and water supply plants in Pearl River Delta

表 1 珠江三角洲湖库采样点编号对应信息

Tab.1 Information of sampling sites of the lakes and reservoirs in Pearl River Delta

\begin{tabular}{|c|c|c|c|c|c|}
\hline 城市 & 编号 & 湖库名称 & 城市 & 编号 & 湖库名称 \\
\hline \multirow[t]{14}{*}{ 广州 } & G1 & 挂绿湖 & \multirow[t]{7}{*}{ 佛山 } & F1 & 云东海北湖 \\
\hline & G2 & 天鹿湖 & & $\mathrm{F} 2$ & 三水南湖 \\
\hline & G3 & 明珠湖 & & F3 & 丹灶仙湖 \\
\hline & G4 & 华农洪泽湖 & & F4 & 秀丽湖 \\
\hline & G5 & 北秀湖 & & F5 & 绿岛湖 \\
\hline & G6 & 荔湾湖 & & F6 & 西樵山天湖 \\
\hline & G7 & 中大北湖 & & F7 & 桂畔湖 \\
\hline & G8 & 海珠湖 & \multirow[t]{4}{*}{ 东莞 } & D1 & 潇湴湖 \\
\hline & G9 & 中心湖 & & D2 & 映泉湖 \\
\hline & G10 & 黄埔湖 & & D3 & 松山湖 \\
\hline & G11 & 莲花山湖 & & D4 & 清溪湖 \\
\hline & G12 & 聚秀湖 & \multirow[t]{3}{*}{ 惠州 } & H1 & 博罗明湖 \\
\hline & G13 & 莲塘坪 & & $\mathrm{H} 2$ & 平湖 \\
\hline & G14 & 白水湖 & & H3 & 红花湖 \\
\hline \multirow[t]{4}{*}{ 肇庆 } & Q1 & 九龙湖 & \multirow[t]{3}{*}{ 深圳 } & $\mathrm{S} 1$ & 龟寿湖 \\
\hline & Q2 & 碧莲湖 & & $\mathrm{S} 2$ & 深圳东湖 \\
\hline & Q3 & 七星湖 & & S3 & 三洲田 \\
\hline & Q4 & 沙田坑 & \multirow[t]{4}{*}{ 珠海 } & P1 & 神泉湖 \\
\hline \multirow[t]{6}{*}{ 中山 } & $\mathrm{Z} 1$ & 得能湖 & & P2 & 白藤湖 \\
\hline & $\mathrm{Z} 2$ & 奥斯湖 & & P3 & 竹仙洞 \\
\hline & $\mathrm{Z3}$ & 紫马岭湖 & & P4 & 翠湖 \\
\hline & $\mathrm{Z} 4$ & 逸仙湖 & \multirow[t]{3}{*}{ 江门 } & $\mathrm{J} 1$ & 江门东湖 \\
\hline & $\mathrm{Z} 5$ & 卓旗山湖 & & $\mathrm{J} 2$ & 天鹅湖 \\
\hline & $\mathrm{Z6}$ & 小琅环湖 & & $\mathrm{J} 3$ & 牛牯石湖 \\
\hline
\end{tabular}




\section{2 实验仪器与试剂}

仪器: 酸度计 (上海精密科学仪器有限公司);78-2 型双向磁力加热搅拌器 (金城国胜实验仪器厂); 程 控六联混凝摚拌仪 (武汉恒岭科技有限公司) ; Agilent 7890A/5975C GC/MSD 气相色谱仪-质谱检测器 (美 国 Agilent 公司); DB-5MS 毛细管柱 $(30 \mathrm{~m} \times 0.25 \mathrm{~mm} \times 0.25 \mu \mathrm{m})$; 50/30 $\mu \mathrm{m} \mathrm{DVB} / \mathrm{CAR} / \mathrm{PDMS}$ 固相微萃取纤 维头.

试剂: 5 种嗅味物质的标准物质均为浓度为 $100 \mu \mathrm{g} / \mathrm{ml}$ 的 $\mathrm{CH}_{3} \mathrm{OH}$ 溶液, 购自上海安谱科学仪器有限公 司; $2.5 \mathrm{mg} / \mathrm{ml}\left(\mathrm{Al}_{2} \mathrm{O}_{3}\right.$ 计) 的 $\mathrm{Al}_{2}\left(\mathrm{SO}_{4}\right)_{3}$ 溶液; $\mathrm{NaCl}$ (分析纯) 使用前经马弗炉 $450^{\circ} \mathrm{C}$ 加热 $4 \mathrm{~h}$ 消除混人的有机 物; $\mathrm{H}_{2} \mathrm{SO}_{4}$ (分析纯) 和 $\mathrm{NaOH}$ (分析纯) 为 $\mathrm{pH}$ 调节剂; $18.2 \mathrm{M} \Omega$ 超纯水, 经 Millipore 公司纯水器制备; 载气为 $99.999 \%$ 高纯度氮气.

\section{3 定性定量方法}

固相微萃取 (Solid Phase MicroExtraction, SPME) 技术具有操作简单、快速和费用低廉等优点 ${ }^{[16-17]}$, GSM 、MIB、TCA、IPMP 和 IBMP 这 5 种嗅味物质均为挥发性或半挥发性有机物 ${ }^{[18-19]}$, 可用 SPME 进行前处 理,并用色谱质谱 (Gas Chromatography/Mass Spectrometry, GC/MS) 进行浓度测定.

前处理方法: 将 $10 \mathrm{ml}$ 水样或标样加人 $20 \mathrm{ml}$ 螺口顶空瓶中, 加人质量分数为 $30 \%$ 的 $\mathrm{NaCl}$ 作为离子强度 调节剂, 用装有聚四氟乙烯硅胶隔垫的螺纹瓶盖盖上, 振荡. 将顶空瓶放置在加热装置中, $60^{\circ} \mathrm{C}$ 预热 $10 \mathrm{~min}$, SPME 萃取头插人顶空瓶内上部空气中萃取 $1000 \mathrm{~s}$, 然后在 GC 进样口 $250^{\circ} \mathrm{C}$ 解吸 $240 \mathrm{~s}$, 再进行 GC 升温程 序测定汽化样品组分 ${ }^{[20]}$.

GC/MS 条件: 高纯氮( $99.999 \%$ ) 载气流量 $1.5 \mathrm{ml} / \mathrm{min}$, 隔垫吹扫流量保持 $3 \mathrm{ml} / \mathrm{min}, \mathrm{GC}$ 进样口温度 $250^{\circ} \mathrm{C}$, 采用不分流模式进样; 色谱柱程序升温: $40^{\circ} \mathrm{C}$ 保持 $2 \mathrm{~min}$, 经 $8^{\circ} \mathrm{C} / \mathrm{min}$ 升至 $165^{\circ} \mathrm{C}$, 保持 $3 \mathrm{~min}$. 传输线 温度 $280^{\circ} \mathrm{C}$. $\mathrm{EI}$ 离子源温度 $230^{\circ} \mathrm{C}$, 四极杆温度 $150^{\circ} \mathrm{C}$, 电子能量 $70 \mathrm{eV}$, 质量数扫描范围 $10 \sim 350 \mathrm{amu}^{[20]}$.

\section{4 质量控制与保证}

每个水样与标样均设置 3 组平行样, 并设置空白样 (吸取 $10 \mathrm{ml}$ 的超纯水, 以同样的步骤进行实验). 使 用标准物质配制浓度为 1 200 $\mathrm{ng} / \mathrm{L}$ 的混合标准溶液, 并用 GC/MS 测定标样. 通过标样的色谱图确定目标 化合物的出峰时间和特征离子, 并得到标准曲线及其决定系数 $\left(R^{2}\right)$ 和精密度 (Relative Standard Deviation, RSD); 根据美国 EPA 的标准, 采用 3 倍信噪比计算各目标物的方法检出限 (Method Detection Limit, MDL), 结果如表 2 所示.

表 2 嗅味物质的出峰时间和特征离子以及 $R^{2} 、 \operatorname{RSD}$ 和 MDL

Tab.2 Appearance time, characteristic ions, $R^{2}$, RSD and MDL of odor and taste compounds

\begin{tabular}{cccccc}
\hline 嗅味物质 & 出峰时间 $/ \mathrm{min}$ & 特征离子 $/(\mathrm{m} / \mathrm{z})$ & $R^{2}$ & $\mathrm{RSD} / \%$ & $\mathrm{MDL} /(\mathrm{ng} / \mathrm{L})$ \\
\hline IPMP & 9.766 & $137,152,124$ & 0.999663 & 3.89 & 0.31 \\
IBMP & 11.451 & $124,151,94$ & 0.999889 & 3.33 & 0.29 \\
MIB & 11.752 & $95,107,43$ & 0.999901 & 3.75 & 0.03 \\
TCA & 14.142 & $210,167,195$ & 0.999422 & 2.35 & 0.02 \\
GSM & 15.667 & $112,125,41$ & 0.999781 & 1.52 & 0.38 \\
\hline
\end{tabular}

由表 2 可知,运用本实验所采用的方法测定这 5 种嗅味物质,得到的标准曲线均具有良好的线性关系， $R^{2}$ 均在 0.999 以上, RSD 均控制在 $5 \%$ 以内, 且该方法对 5 种嗅味物质的 MDL 均较低. 而且, 空白样中各嗅味 物质的检测结果均低于其检出限. 因此,采用此分析方法可以准确有效地检测水体中嗅味物质的浓度.

\section{2 结果与分析}

\section{1 珠江三角洲湖库水体嗅味物质浓度}

使用上述方法对珠江三角洲 9 个城市的湖库水体中的 5 种嗅味物质进行浓度检测, 得到各湖库中 5 种 嗅味物质的总浓度, 结果见图 2. 由图 2 可知, 珠江三角洲 9 个城市的 48 个湖库水体中均有嗅味物质检出, 说明嗅味物质已经普遍存在于珠江三角洲的湖库水体中. 其中, 广州、佛山、肇庆、东莞、惠州和深圳 6 个城 


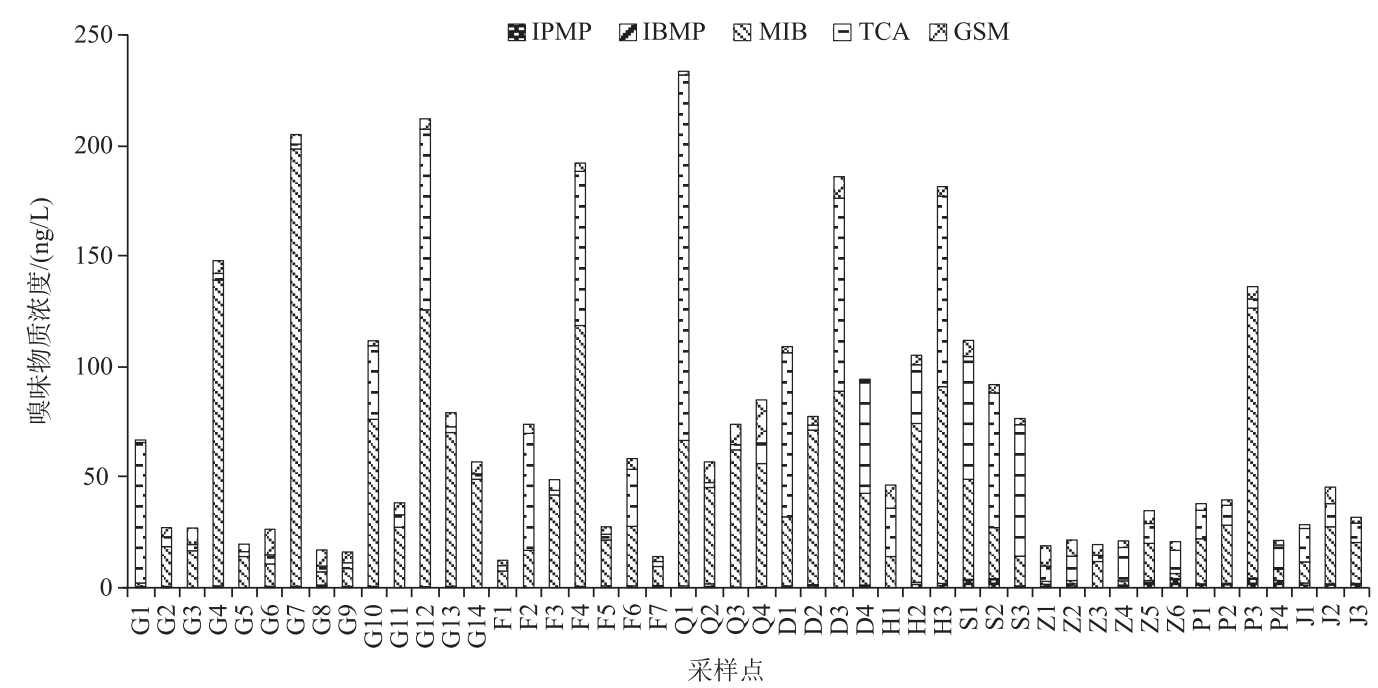

图 2 珠江三角洲湖库水体中嗅味物质浓度检测结果

Fig. 2 Test results of odor and taste compounds in water samples from lakes and reservoirs in Pearl River Delta

市的湖库水体中嗅味物质浓度较高, 其嗅味物质平均浓度分别为 $71.01 、 70.93 、 102.37 、 116.61 、 110.96$ 和 $93.38 \mathrm{ng} / \mathrm{L}$. 广州的华农洪泽湖、中大北湖、黄埔湖和聚秀湖、佛山的秀丽湖、肇庆的九龙湖、东莞的松山湖、 惠州的平湖和红花湖以及深圳的龟寿湖等, 其嗅味物质总浓度均超过了 $100.00 \mathrm{ng} / \mathrm{L}$. 而中山、珠海与江门 3 市的湖库水体中嗅味物质浓度相对较低, 其平均浓度分别为 $22.78 、 58.82$ 和 $35.22 \mathrm{ng} / \mathrm{L}$. 除了珠海的竹仙洞 水体中嗅味物质总浓度为 $136.20 \mathrm{ng} / \mathrm{L}$ 外,这 3 个城市其余湖库的嗅味物质总浓度均低于 $50.00 \mathrm{ng} / \mathrm{L}$. 导致 水体产生异嗅味的生物主要有放线菌、㩰类和真菌 ${ }^{[20-22]}$. 当水源中某些藻类大量繁殖时, 在其新陈代谢过程 中会分泌多种嗅味物质, 从而导致水体变臭. 因为此次采样时间为枯季, 各湖库补给河流的流量较小甚至无 河流汇人, 再加上湖库一般呈封闭或半封闭状态, 其水体置换功能较弱, 从而有利于藻类的繁殖, 进而导致 了部分湖库的嗅味物质浓度较高. 此外, 广州、佛山、肇庆、东莞、惠州和深圳 6 个城市的嗅味物质浓度较高, 可能与汇人其中的营养物质浓度较高有关.

为了更全面地了解珠江三角洲湖库水体中嗅味物质的污染情况, 本文进一步对各湖库水体中的 5 种嗅 味物质所占的比重进行分析, 结果见图 3. 图 3 表明, MIB、TCA 和 GSM 是珠江三角洲湖库中的主要嗅味物 质,这 3 种嗅味物质占了嗅味物质总浓度的 $80.18 \% \sim 100.00 \%$. 其中, 在广州的挂绿湖、佛山的三水南湖、肇 庆的九龙湖、东莞的潇湴湖和清溪湖、深圳的东湖和三洲田、中山的逸仙湖、珠海的翠湖以及江门的东湖这 10 个湖库中, TCA 的相对含量最高, 占到了这些湖库的嗅味物质总浓度的 50\%以上; 广州的荔湾湖和海珠湖 以及中山的得能湖这 3 个湖库水体的主要嗅味物质为 GSM; 而在佛山的西樵山天湖、东莞的松山湖、惠州的 博罗明湖和红花湖以及深圳的龟寿湖这 5 个湖库中, MIB 和 TCA 分别占了各湖库水体嗅味物质总浓度的 $40 \%$ 以上; 除此之外的 30 个湖库 (占了湖库总数量的 $62.50 \%$ ), 其水体中的主要嗅味物质均为 MIB. 不同的 藻类产生的主要嗅味物质是不同的. Sugiura 等 ${ }^{[23]}$ 的研究发现 MIB、GSM 和 TCA 主要由蓝绿藻和硅藻产生, 施择等 ${ }^{[24]}$ 和王敬富等 ${ }^{[25]}$ 的研究表明在冬季我国南方湖库中的藻类以蓝藻门、绿藻门和硅澡门为主. 另有研 究表明, 硅藻喜较低温度 ${ }^{[26-27]}$ 、且对光照强度的适应能力较强 ${ }^{[28]}$, 蓝藻门中的颤藻属较能适应低光强 ${ }^{[29]}$. 同 时, 根据肖作义等 ${ }^{\left[{ }^{[0]}\right.}$ 和丁震 ${ }^{\text {洔 }^{[31]}}$ 的研究, MIB 主要由颤藻属产生. 因此, 珠江三角洲湖库水体中的嗅味物质 可能主要来源于水体中的硅藻门和蓝藻门中的颤藻属.

\section{2 污水处理厂对嗅味物质的去除效果分析}

珠江三角洲的污水处理厂 $\mathrm{A}$ 和 $\mathrm{B}$ 所处理的均为城镇居民生活污水, 污水处理厂 $\mathrm{A}$ 采用的是氧化沟工 艺, 污水处理厂 $\mathrm{B}$ 采用的是厌氧/缺氧/耗氧 $\left(\mathrm{A}^{2} / 0\right)$ 工艺. 本研究对这 2 个污水处理厂工艺流程中的嗅味物 


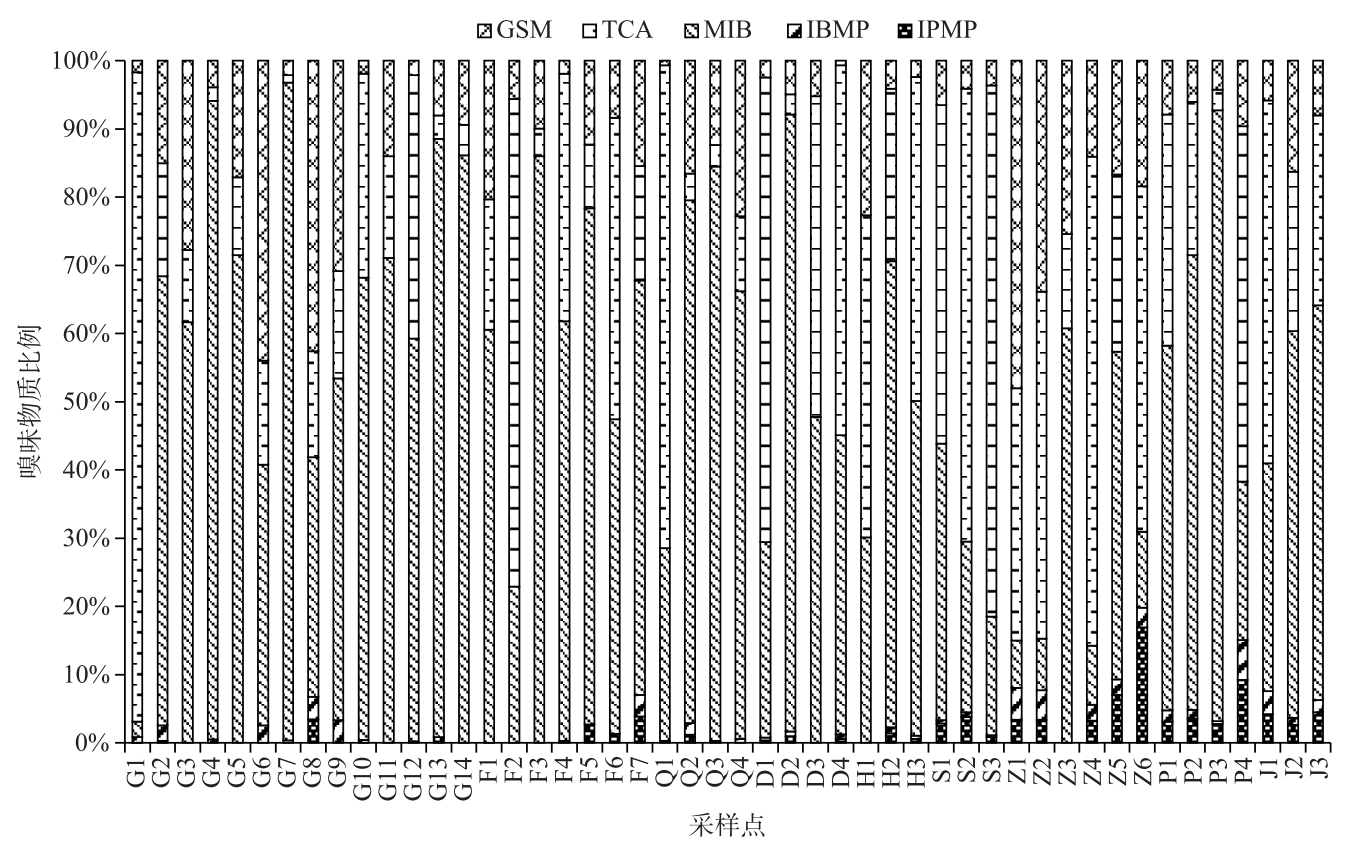

图 3 珠江三角洲湖库水体中 5 种嗅味物质的比例

Fig.3 Proportions of 5 odor and taste compounds in water samples from lakes and reservoirs in Pearl River Delta 质浓度进行检测分析, 其结果见图 4.

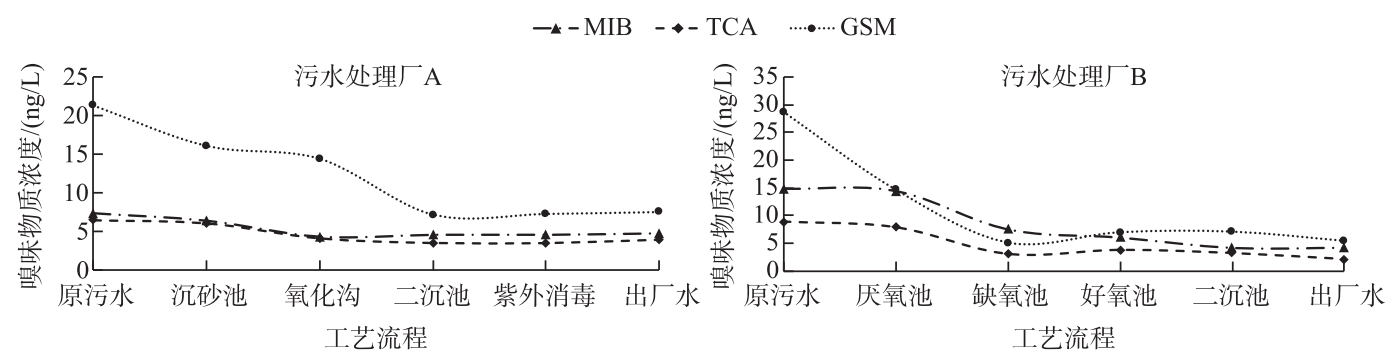

图 4 污水处理厂 $\mathrm{A}$ 和 $\mathrm{B}$ 中嗅味物质的浓度变化趋势

Fig.4 Concentrations changing trends of odor and taste compounds in wastewater treatment plants A and B

经过了污水处理厂的各级处理工艺, MIB、TCA 和 GSM 这 3 种嗅味物质的浓度都有所降低. 污水处理厂 $\mathrm{A}$ 和 B 对 MIB 的去除率分别为 $35.33 \%$ 和 $71.78 \%$, 平均为 $53.55 \%$; 对 TCA 的去除率分别为 $39.15 \%$ 和 $75.65 \%$, 平均为 $57.40 \%$; 对 GSM 的去除率分别为 $64.58 \%$ 和 $81.21 \%$, 平均为 $72.90 \%$. 采用 $\mathrm{A}^{2} / 0$ 工艺的污水 处理厂 $\mathrm{B}$ 对嗅味物质的去除效果明显优于采用氧化沟工艺的污水处理厂 $\mathrm{A}$ (图 5). 这可能是由于 $\mathrm{A}^{2} / \mathrm{O}$ 工 艺将厌氧池、缺氧池和耗氧池分开, 厌氧、缺氧和耗氧 3 种不同的环境条件和不同种类的微生物菌群, 能够 更有效地去除水体中的嗅味物质. 在污水处理厂 $\mathrm{A}$ 中, 除了 $\mathrm{A}^{2} / \mathrm{O}$ 主体工艺外, 由于曝气沉砂池在曝气过程 中将挥发性或半挥发性的嗅味物质随气泡一起去除 ${ }^{[18-19]}$, 因此污水经过曝气沉砂池后其嗅味物质浓度亦有 一定的下降.

\section{3 自来水厂对嗅味物质的去除效果分析}

珠江三角洲河网密集, 许多湖库与河道作为自来水厂的重要水源地, 为人们的生活用水和工业用水等 提供保障. 本研究对珠江三角洲的自来水厂 C 和 $\mathrm{D}$ 的水处理流程中的嗅味物质进行检测分析, 两个自来水 
厂均采用混凝、沉淀和过滤等水处理工艺. 水厂的混凝沉淀等工艺能够对水体中嗅味物质浓度产生一定的 影响 ${ }^{[20]}$. 自来水厂 C 和 D 的各级处理工艺中 MIB、TCA 和 GSM 这 3 种嗅味物质的浓度变化情况见图 5.

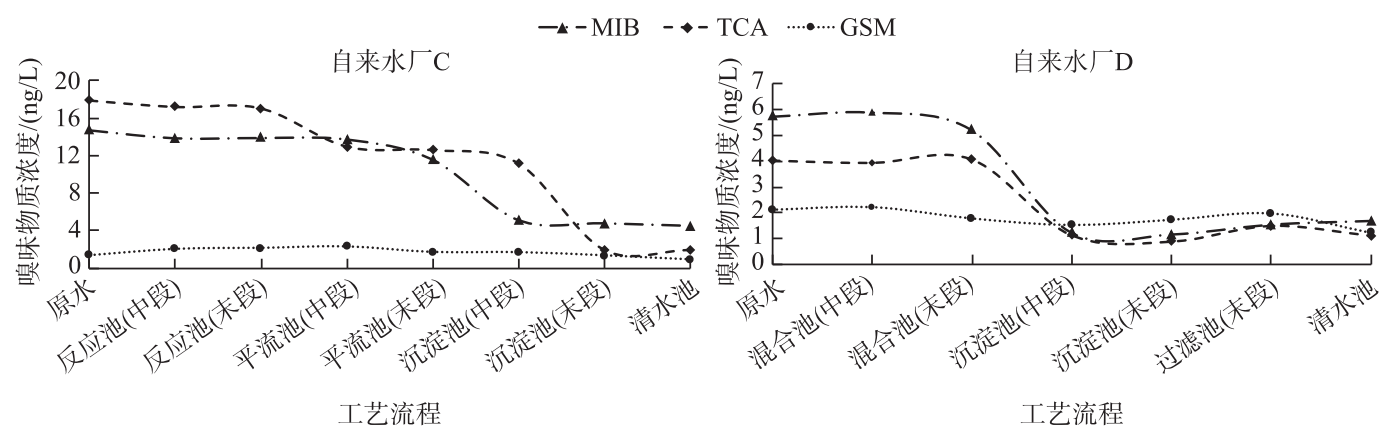

图 5 自来水厂 $\mathrm{C}$ 和 $\mathrm{D}$ 中嗅味物质的浓度变化趋势

Fig.5 Concentrations changing trends of odor and taste compounds in water supply plants C and D

自来水厂中的嗅味物质浓度沿着水处理工艺流程的大致趋势是下降的. 经过各级水处理工艺后, 自来 水厂 $\mathrm{C}$ 和 D 对 MIB 的去除率分别为 57.79\% 和 70.50\%, 平均为 $64.14 \%$; 对 TCA 的去除率分别为 $67.20 \%$ 和 $72.07 \%$, 平均为 $69.63 \%$; 对 GSM 的去除率分别为 $32.19 \%$ 和 $41.54 \%$, 平均为 $36.86 \%$ ( 图 5).

虽然常规水处理工艺对嗅味物质的去除效果比较不稳定 ${ }^{[32]}$, 但从图 5 可以看出, 混凝沉淀工艺对 MIB、 TCA 和 GSM 这 3 种嗅味物质具有一定的去除效果. 混凝沉淀工艺对嗅味物质的去除主要有以下 2 个原因: 一是嗅味物质属于挥发性或挥发性有机物 ${ }^{[18-19]}$, 通过混凝摚拌会使其挥发而造成一定的损失; 二是由于自 来水厂 C 和 D 的混凝剂均为聚合氯化铝, 聚合氯化铝属于无机高分子絮凝剂 ${ }^{[33]}$, 能够对嗅味物质等有机物 进行裹挟去除.

\section{4 强化混凝沉淀工艺对嗅味物质的去除效果分析}

为了更好地去除水体中的嗅味物质,本文研究了强化混凝沉淀工艺对嗅味物质的去除效果. 混凝沉淀 工艺对有机物的去除效果受到有机物含量、混凝剂含量、搅拌强度和 $\mathrm{pH}$ 值等的影响 ${ }^{[34]}$. 因此, 本文分别考 虑了混凝剂投加量、嗅味物质初始浓度、反应体系 $\mathrm{pH}$ 值以及搅拌速率对强化混凝沉淀工艺去除 MIB、TCA 和 GSM 的影响.

2.4.1 混凝剂投加量和嗅味物质浓度的影响 第 1 部分:用超纯水配制 $1000 \mathrm{ml}$ 浓度为 $200 \mathrm{ng} / \mathrm{L}$ 的嗅味物质 混合溶液, 分别投人 $0 \sim 8 \mathrm{ml} 2.5 \mathrm{mg} / \mathrm{ml}\left(\mathrm{Al}_{2} \mathrm{O}_{3}\right.$ 计 $)$ 的 $\mathrm{Al}_{2}\left(\mathrm{SO}_{4}\right)_{3}$ 溶液, 300 转 $/ \mathrm{min}$ 快速搅拌 $30 \mathrm{~s}, 100$ 转/ $\mathrm{min}$ 中速搅拌 $7 \mathrm{~min}, 50$ 转 $/ \mathrm{min}$ 慢速搅拌 $1 \mathrm{~min}$; 静置沉淀 $20 \mathrm{~min}$. 取上清液检测各水样的嗅味物质浓度, 结果见 图 6.

第 2 部分: 用超纯水配制 $1000 \mathrm{ml}$ 浓度分别为 $20 \sim 300 \mathrm{ng} / \mathrm{L}$ 的嗅味物质混合溶液,分别投加 $5.5 \mathrm{ml} 2.5$ $\mathrm{mg} / \mathrm{ml}\left(\mathrm{Al}_{2} \mathrm{O}_{3}\right.$ 计 $)$ 的 $\mathrm{Al}_{2}\left(\mathrm{SO}_{4}\right)_{3}$ 溶液, 300 转 $/ \mathrm{min}$ 快速搅拌 $30 \mathrm{~s}, 100$ 转 $/ \mathrm{min}$ 中速搅拌 $7 \mathrm{~min}, 50$ 转 $/ \mathrm{min}$ 慢速 搅拌 $1 \mathrm{~min}$; 静置沉淀 $20 \mathrm{~min}$. 取上清液检测各水样的嗅味物质浓度,结果见图 7.

在 $\mathrm{Al}_{2} \mathrm{O}_{3}$ 投加量较小 (小于 $13.75 \mathrm{mg} / \mathrm{L}$ ) 的情况下, 嗅味物质的去除率随着 $\mathrm{Al}_{2} \mathrm{O}_{3}$ 投加量的增加而增大; 当 $\mathrm{Al}_{2} \mathrm{O}_{3}$ 投加量较大 (大于 $13.75 \mathrm{mg} / \mathrm{L}$ ) 时, 嗅味物质的去除率基本稳定 (图 6). 由此可见, $\mathrm{Al}_{2} \mathrm{O}_{3}$ 混凝剂对嗅 味物质的去除率有一个临界值, 当 $\mathrm{Al}_{2} \mathrm{O}_{3}$ 投加量大于临界值时, 体系达到饱和, 增大投加量对嗅味物质的去 除无明显的促进作用. 当嗅味物质的初始浓度为 $200 \mathrm{ng} / \mathrm{L}$ 时, $\mathrm{Al}_{2} \mathrm{O}_{3}$ 投加量的临界值约为 $13.75 \mathrm{mg} / \mathrm{L}$. 体系 稳定后, $\mathrm{Al}_{2} \mathrm{O}_{3}$ 对 TCA、GSM 和 MIB 的去除率分别为 60.23\%、58.55\% 和 44.44\%. 由于在 $\mathrm{Al}_{2} \mathrm{O}_{3}$ 对有机物的去 除过程中, 主要依靠铝盐的吸附架桥和网捕卷扫作用 ${ }^{[35]}$, 当 $\mathrm{Al}_{2} \mathrm{O}_{3}$ 投加量大于临界值时, 会产生 “胶体保 护”, 使脱稳胶粒电荷变号或使胶粒被包卷重新稳定 ${ }^{[36]}$, 故体系饱和后增大 $\mathrm{Al}_{2} \mathrm{O}_{3}$ 投加量无法提高嗅味物质 的去除率.

当 $\mathrm{Al}_{2} \mathrm{O}_{3}$ 投加量一定 $(13.75 \mathrm{mg} / \mathrm{L})$ 时, 随着嗅味物质初始浓度的增大, $\mathrm{Al}_{2} \mathrm{O}_{3}$ 对嗅味物质的去除率逐渐 降低并趋于稳定 (图 7). 说明定量的 $\mathrm{Al}_{2} \mathrm{O}_{3}$ 仅能去除一定量的嗅味物质, 当嗅味物质浓度较大时, 需要增大 


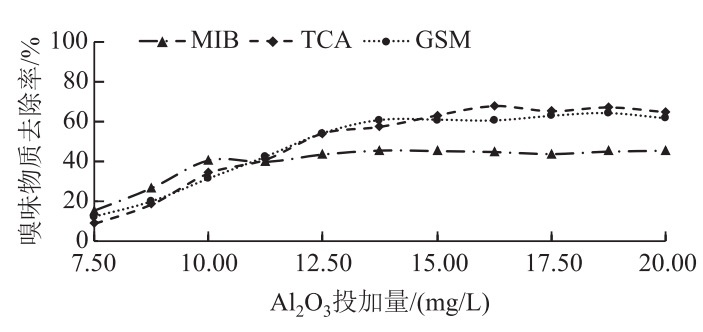

图 6 混凝剂投加量对嗅味物质去除率的影响

Fig.6 Effect of coagulant dosage on removal rates of odor and taste compounds

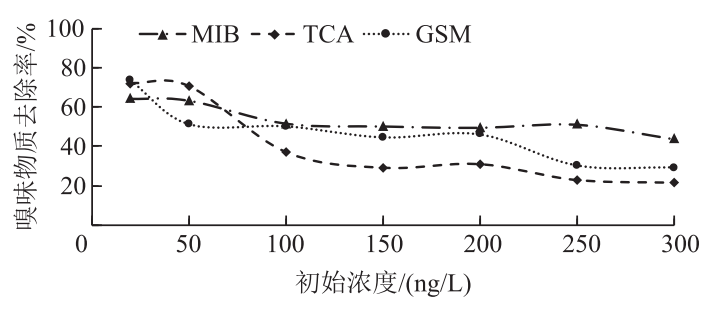

图 7 嗅味物质初始浓度对其去除率的影响

Fig.7 Effect of odor and taste compounds' initial concentrations on their removal rates

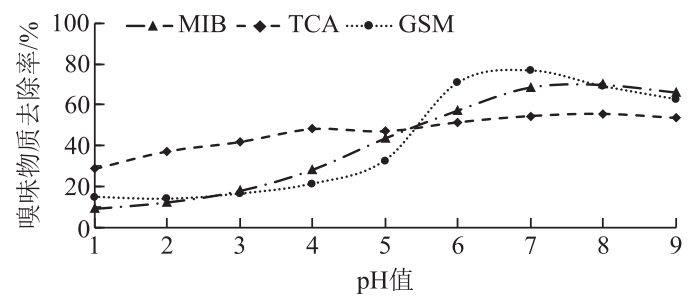

图 $8 \mathrm{pH}$ 值对嗅味物质去除率的影响

Fig. 8 Effect of $\mathrm{pH}$ value on removal rates of odor and taste compounds
$\mathrm{Al}_{2} \mathrm{O}_{3}$ 投加量方可有效地去除嗅味物质.

因此, $\mathrm{Al}_{2} \mathrm{O}_{3}$ 投加量对嗅味物质的去除有重要作 用, 但其投加量存在临界值. $\mathrm{Al}_{2} \mathrm{O}_{3}$ 投加量过低, 无法 形成凝聚, 对嗅味物质的去除率较低; $\mathrm{Al}_{2} \mathrm{O}_{3}$ 投加量 过高, 不仅会造成浪费和增加水处理的成本, 而且会 使多余的铝盐造成二次污染.

$2.4 .2 \mathrm{pH}$ 值的影响 用超纯水配制 $1000 \mathrm{ml}$ 浓度为 $200 \mathrm{ng} / \mathrm{L}$ 的嗅味物质混合溶液, 将其 $\mathrm{pH}$ 值分别调 节至 1 9, 分别投加 $5.5 \mathrm{ml} 2.5 \mathrm{mg} / \mathrm{ml}\left(\mathrm{Al}_{2} \mathrm{O}_{3}\right.$ 计) 的 $\mathrm{Al}_{2}\left(\mathrm{SO}_{4}\right)_{3}$ 溶液, 300 转/ $\mathrm{min}$ 快速搅拌 $30 \mathrm{~s}, 100$ 转/ $\min$ 中速摚拌 $7 \mathrm{~min}, 50$ 转 $/ \mathrm{min}$ 慢速搅拌 $1 \mathrm{~min}$; 静 置沉淀 $20 \mathrm{~min}$, 取上清液检测各水样的嗅味物质 浓度.

从图 8 可以看出, 反应体系的酸碱度对嗅味物 质的去除率有着较为重要的影响. 随着反应体系中 $\mathrm{pH}$ 值的增大, 混凝沉淀对水体中嗅味物质的去除 率先升高后降低. 当 $\mathrm{pH}$ 值为 7 时, GSM 的去除率达 到最优, 为 $76.74 \%$; 当 $\mathrm{pH}$ 值为 8 时, MIB 的去除率 达到最优, 为 $74.92 \%$; 当 $\mathrm{pH}$ 值为 6 时, TCA 的去除 率达到最优, 为 $51.46 \%$. 因此, 当 $\mathrm{pH}$ 值处于 $5 \sim 8$ 的 范围内时, 混凝沉淀对嗅味物质的去除率均大于强 酸性 $(\mathrm{pH}<4)$ 与强碱性 $(\mathrm{pH}>9)$ 条件下的去除率. 因 为水体中的嗅味物质是憎水性有机物, 在水中以分 子形态存在且不会在水中发生电离, 因此 $\mathrm{pH}$ 值不 会影响嗅味物质在水中的存在形态. 由此可知, $\mathrm{pH}$ 值对嗅味物质去除率的影响主要是通过影响铝盐 混凝剂的性质来实现的. 当 $\mathrm{pH}$ 过低时, 混凝剂的水 解受到限制, 使得体系中没有足够的差基 $(-\mathrm{OH})$ 进行桥联作用, 絮凝效果较差; 当 $\mathrm{pH}$ 值过高时, 混 凝剂又会溶解生成带负电荷的络合离子而不能很 好地发挥混凝作用 ${ }^{[37-38]}$. 因此, $\mathrm{pH}$ 值过低或过高均 会造成混凝处理效果较差, 使得嗅味物质的去除率 不高.

2.4 .3 搅拌强度的影响 用超纯水配置 $1000 \mathrm{ml}$ 浓度为 $200 \mathrm{ng} / \mathrm{L}$ 的嗅味物质混合溶液, 分别投加 $5.5 \mathrm{ml}$ $2.5 \mathrm{mg} / \mathrm{ml}\left(\mathrm{Al}_{2} \mathrm{O}_{3}\right.$ 计 $)$ 的 $\mathrm{Al}_{2}\left(\mathrm{SO}_{4}\right)_{3}$ 溶液, 分别在 $50 、 100$ 和 300 转 $/ \mathrm{min}$ 的不同搅拌强度下摚拌 $14 \mathrm{~min}$, 静置 沉淀 $20 \mathrm{~min}$,取上清液检测各水样的嗅味物质浓度.

因为机械搅拌是简单、经济和高效的混凝反应摚拌方式 ${ }^{[39]}$,所以本研究用不同的搅拌强度来表示不同 的水力条件. 图 9 反映了不同搅拌强度 (即水力条件) 对嗅味物质去除效果的影响.

在慢速搅拌 (50 转/ min) 的条件下, 嗅味物质的去除率在摚拌初始阶段是较低的, 当搅拌时间达到 $12 \mathrm{~min}$ 之后, 嗅味物质的去除率才基本稳定 (图 9a). 在中速搅拌 (100 转/ min) 的条件下,嗅味物质的去除率在搅拌时 间达到 $8 \mathrm{~min}$ 后才稳定 (图 $9 \mathrm{~b}$ ). 在快速搅拌 (300 转/ $\mathrm{min}$ ) 的条件下, 嗅味物质的去除率只需 $4 \mathrm{~min}$ 便达到稳定 (图 9c) ; 而且相对于慢速搅拌和中速搅拌, 快速搅拌对嗅味物质的去除率明显增大, 约为 $50 \%$. 因此, 搅拌速率 的大小对嗅味物质的去除率有重要影响. 搅拌速率越快, 嗅味物质的去除率越高且越快达到稳定. 

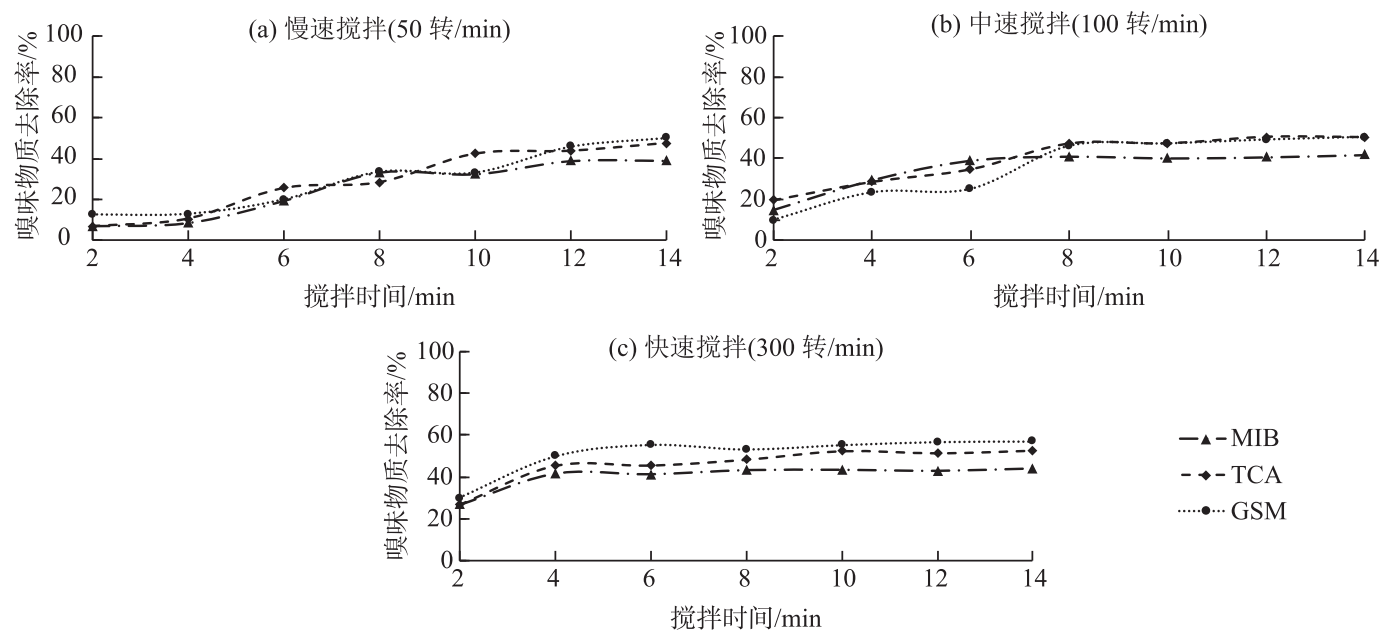

图 9 搅拌强度对嗅味物质去除率的影响

Fig.9 Effect of stirring intensity on removal rates of odor and taste compounds

\section{3 结论}

1) 珠江三角洲 9 个城市的 48 个湖库水体中嗅味物质的检出率达到 $100 \%$, 说明嗅味物质已经普遍存在 于珠江三角洲的湖库水体中. 其中, 广州、佛山、肇庆、东莞、惠州和深圳 6 市的湖库水体中嗅味物质浓度较 高, 而中山、珠海与江门 3 市的湖库水体中嗅味物质浓度相对较低. MIB、TCA 和 GSM 是珠江三角洲湖库水 体中的主要嗅味物质,这 3 种嗅味物质浓度占嗅味物质总浓度的 $80.18 \% \sim 100.00 \%$.

2) 污水处理厂和自来水厂的处理工艺对嗅味物质有一定的去除效果, 嗅味物质的去除主要发生在混凝 沉淀阶段.

3) $\mathrm{Al}_{2} \mathrm{O}_{3}$ 投加量对嗅味物质的去除存在临界值, 当嗅味物质初始浓度为 $200 \mathrm{ng} / \mathrm{L}$ 时, $\mathrm{Al}_{2} \mathrm{O}_{3}$ 投加量为 $13.75 \mathrm{mg} / \mathrm{L}$ 可使得混凝沉淀工艺对嗅味物质的去除效果最佳且铝盐不超标. 当反应体系的 $\mathrm{pH}$ 值为 $5 \sim 8$ 时, 混凝沉淀工艺对嗅味物质的去除效果最佳; $\mathrm{pH}$ 值过低或过高均会造成混凝处理效果较差, 使得嗅味物 质的去除率不高. 搅拌速率的快慢对嗅味物质的挥发和混凝沉淀的反应速率均有一定的影响, 进而对嗅味 物质的去除率造成影响;搅拌速率越快,嗅味物质的去除率越高且越快达到稳定.

\section{4 参考文献}

[ 1 ] Chen WJ. Study on variation and removal of organics during the process of pre-pressure and coagulation sedimentation treatment [Dissertation]. Yangzhou: Yangzhou University, 2015. [ 陈雯婧. 预压力混凝沉淀除藻工艺中有机物变化规律 与去除研究 [学位论文]. 扬州: 扬州大学, 2015.]

[ 2 ] Yu JW, Li ZL, Cao N et al. Analyses of cause for odor and potential problems in water source during odor episode event in Wuxin. Acta Sci Circum, 2007, 27(11): 1771-1777. [于建伟, 李宗来, 曹楠等. 无锡市饮用水嗅味突发事件致嗅原 因及潜在问题分析. 环境科学学报, 2007, 27(11): 1771-1777.]

[ 3 ] Li L, Wan N, Gan NQ et al. Annual dynamics and origins of the odorous compounds in the pilot experimental area of Lake Dianchi, China. Water Sci Technol, 2007, 55(5) : 43-50.

[ 4 ] Zuo Y, Li L, Zhang T et al. Contribution of Streptomyces in sediment to earthy odor in the overlying water in Xionghe Reservoir, China. Water Res, 2010, 44(20): 6085-6094.

[ 5 ] Liang CZ, Wang DS, Sang YM et al. Optimization of determination method of earthy-musty odorants in drinking water. China Water Wastewater, 2007, 23(10): 76-78. [梁存珍, 王东升, 桑义敏等. 饮用水中土霉味物质测定方法的优 化. 中国给水排水, 2007, 23(10): 76-78.] 
[6] Lu NN, Jia RB, Sun SH et al. Research progress of odor material of algae in drinking water sources. Water Wastewater $E n g, 2012,48$ (S2) : 93-95. [逯南南, 贾瑞宝, 孙韶华等. 饮用水中藻源臭味物质的研究进展. 给水排水, 2012, 48(S2): 93-95.]

[ 7 ] Liu ZF, Zhang SQ, Zhuo WS et al. Research of odor compounds and toxicity in the surface water of Haizhu District Guangzhou. J Lake Sci, 2013, 25(6) : 900-906. DOI: 10.18307/2013.0614. [刘祖发, 张素琼, 卓文珊等. 广州市海珠区地 表水中致嗅物质及毒性分析. 湖泊科学, 2013, 25(6): 900-906.]

[ 8 ] Yang ZS, Lu M, Yuan DX et al. Pilot study on odor removal in Kinmen Waterworks. Water Wastewater Eng, 2008,34 (5)：18-22. [杨忠盛, 芦敏, 袁东星等. 金门水厂原水中臭味物质去除的中试研究. 给水排水, 2008, 34 (5): 18-22.]

[ 9 ] Tung SC, Lin TF, Yang FC et al. Seasonal change and correlation with environmental parameters for 2-MIB in Feng-Shen Reservoir, Taiwan. Environ Monit Assess, 2008, 145(1/2/3) : 407-416.

[10] Chiu YT, Yen HK, Lin TF. An alternative method to quantify 2-MIB producing cyanobacteria in drinking water reservoirs: Method development and field applications. Environ Res, 2016, 151: 618-627.

[11] Westerhoff P, Rodriguezhernandez M, Baker L et al. Seasonal occurrence and degradation of 2-methylisoborneol in water supply reservoirs. Water Res, 2005, 39(20): 4899-4912.

[12] Liang H, Li GB, Li X et al. Effect of water treatment process selection on algae removal efficiency. China Water Wastewater, 2005, 21(3) : 5-7. [梁恒, 李圭白, 李星等. 不同水处理工艺流程对除藻效果的影响. 中国给水排水, $2005,21(3): 5-7$.

[13] Hageskal G, Lima N, Skaar I. The study of fungi in drinking water. Mycol Res, 2009, 113(2) : 165-172.

[14] Liu C, Gao NY, Ma XY. Study on emergent treatment technologies used in algae-laden and microcystins pollution raw water. Chin Water Wastewater, 2006, 22(21): 9-13. [刘成, 高乃云, 马晓雁. 高藻及微囊藻毒素污染原水的应急处理 工艺研究. 中国给水排水, 2006, 22(21): 9-13.]

[15] Tan M, Liu K, Liu L et al. Spatialization of population in the Pearl River Delta in $30 \mathrm{~m}$ grids using random forest model. Prog Geo, 2017, 36(10): 1304-1312. [谭敏, 刘凯, 柳林等. 基于随机森林模型的珠江三角洲 $30 \mathrm{~m}$ 格网人口空间 化. 地理科学进展, 2017, 36(10): 1304-1312.]

[16] Mester Z, Sturgeon R. Trace element speciation using solid phase microextraction. Spectrochim Acta B, 2005, 60(9): 1243-1269.

[17] Qiao MS, Zhao SX, Liang HZ et al. Quantification of two components in Chinese liquor by SPME-GC-MS. Food Sci, 2015, 36(16) : 224-227. [乔敏莎, 赵树欣, 梁慧珍等. 固相微萃取-GC-MS 定量检测白酒中两种异嗅物质. 食品科 学, 2015, 36(16): 224-227.]

[18] Ma XY, Gao NY, Li QS et al. Determination of trace Geosmin and 2-Methylisoborneol in drinking water by gas chromatography. J Instr Anal, 2007, 26(2) : 267-269. [马晓雁, 高乃云, 李青松等. 气相色谱法测定饮用水中痕量土臭素和 二甲基异冰片. 分析测试学报, 2007, 26(2) : 267-269.]

[19] Li FA, Zhang HW, Chao M et al. Determining Geosmin and 2-Methylisoborneol by solid phase extraction-gas chromatography. Water Technol, 2011, 5(2): 54-55. [李福安, 张焕伟, 巢猛等. 固相萃取-气相色谱法测定土臭素和 2-甲基异 冰片. 供水技术, $2011,5(2): 54-55$.

[20] Liu ZF, Chen JC, Zhuo WS et al. Degradation effects of odor and taste compounds in water plants in a city of Pearl River Delta. J Subtrop Resour Environ, 2017, 12(2): 1-7. [刘祖发, 陈记臣, 卓文珊等. 珠江三角洲某市水厂中异嗅物质 的降解效果研究. 亚热带资源与环境学报, 2017, 12(2): 1-7.]

[21] Li L, Chen W, Song LR. Extermination of earthy/musty odorous metabolites in lakes and ponds in China. Abstracts of 5th Asia Pacific Conference on Algal Biotechnology, 2003.

[22] Sun W, Gong XL, Chen Y et al. Photochemical degradation of the algae-derived dissolved organic matter in Lake Taihu. $J$ Lake Sci, 2018, 30(1) : 91-101. DOI: 10.18307/2018.0109. [孙伟, 巩小丽, 陈爆等. 太湖藻源溶解性有机质光化 学降解研究. 湖泊科学, 2018, 30(1): 91-101.]

[23] Sugiura N, Yagi O, Sudo R. Musty odor from blue-green alga, Phormidium tenue in Lake Kasumigaura. Environ Technol, 1986, 7(1): 77-86.

[24] Shi Z, Li AJ, Zhang YX et al. Research on phytoplankton community of Dianchi Lake. Environ Monit Chin, 2014,30 (5)：121-124. [施择, 李爱军, 张榆霞等. 滇池浮游藻类群落构成调查. 中国环境监测, 2014, 30(5): 121-124.] 
[25] Wang JF, Chen JA, Li QH et al. Spatial distribution and recruitment simulation test of over-winter algae in Lake Hongfeng, Guizhou. J Lake Sci, 2013, 25(1) : 108-114. DOI: 10.18307/2013.0114. [王敬富, 陈敬安, 李秋华等. 贵州红 枫湖越冬藻类的空间分布与实验室复苏实验. 湖泊科学, 2013, 25(1) : 108-114.]

[26] Wang SF. The means of removing algae in lake water. Pollut Control Technol, 2000, 13(1): 23-25. [王士芬. 湖泊水藻 类的去除方法. 污染防治技术, 2000, 13(1) : 23-25.]

[27] Welch EB, Lindell T eds. Ecological effects of waste water. Cambridge: Cambridge University Press, 1980.

[28] Foudy B ed. Phycology. Shanghai: Shanghai Scientific \& Technical Publishers, 1980. [B · 福迪. 藻类学. 上海: 上海科 学技术出版社, 1980.]

[29] Wu L, Liu XH, Cheng XY et al. Spatial-temporal variation of phytoplankton in clean landscape water in Southeast China from Autumn to Spring and analysis of the related factors. Environ Sci Technol, 2012, 35(4): 120-126. [武琳, 刘雪华, 成小英等. 景观水体浮游藻类变化及与水质因子关系分析. 环境科学与技术, 2012, 35(4): 120-126.]

[30] Xiao ZY, Yang BW, Yang D et al. The characters of algae in a reservoir water from Yellow River in winter. Water Technol, 2009, 3(6) : 6-9. [ 肖作义, 杨炳武, 杨棣等. 冬季黄河水源水库中藻类变化特征研究. 供水技术, 2009, 3(6): 6-9.]

[31] Ding Z. Identification, distribution and treatment of algae-producing odorous compounds in water [Dissertation]. Nanjing: Southeast University, 2017. [丁震. 水体中藻源致嗅物质的辨识、分布与处理研究 [学位论文]. 南京: 东南大 学, 2017.]

[32] Bruce D, Westerhoff P, Brawley-Chesworth A. Removal of 2-methylisoborneol and geosmin in surface water treatment plants in Arizona. J Water Supply Res T, 2002, 51(4) : 183-197.

[33] Tang HX. Basic studies of inorganic polymer flocculants. Environ Chem, 1990, 9(3): 1-12. [汤鸿霄. 无机高分子絮凝 剂的基础研究. 环境化学, 1990, 9(3): 1-12.]

[34] Zhuo WS, Zhang LJ, Liu ZF et al. The research on enhanced coagulation controlling organic matter and residual aluminum in water. Acta Sci Nat Univ Sunyatseni, 2012, 51(4): 129-133. [卓文珊, 张丽娟, 刘祖发等. 强化混凝控制有机物 和残余铝的试验研究. 中山大学学报: 自然科学版, 2012, 51(4) : 129-133.]

[35] Liu XX, Yang ZL, Gao BY et al. Coagulation characteristics of polymeric chloride-poly (epichlorohydrin-dimethylamine) composite flocculant for simulated water treatment. Environ Sci, 2013, 34(9): 3493-3501. [刘新新, 杨忠莲, 高宝玉 等. 聚合氯化铁-聚 (环氧氯丙烷-二甲胺)复合絮凝剂在模拟水处理中的混凝特性研究. 环境科学, 2013, 34(9): 3493-3501.]

[36] Shi WY, Sheng L, Jiang ZF. Study on the effect of dosage and $\mathrm{pH}$ on the removal of organic matter by coagulation. J Jilin Radio TV Univ, 2008, (1) : 103-105. [石文扬, 盛力, 姜志飞. 加药量与 $\mathrm{pH}$ 值对强化混凝去除有机物的影响研究. 吉林广播电视大学学报, 2008, (1) : 103-105.]

[37] Pang ZD. Study on the water resources of coal mine: A case study of Huainan Mining Area [Dissertation]. Huainan: Anhui University of Science and Technology, 2005. [ 庞振东. 煤矿矿井水资源化研究一一以淮南矿区为例 [ 学位论文]. 淮南: 安徽理工大学, 2005.]

[38] Zhong Q. Study on the treatment of electrolytical manganese wastewater [Dissertation]. Changsha: Hunan University, 2006. [钟琼. 电解锰生产废水处理技术的研究 [ 学位论文]. 长沙: 湖南大学, 2006.]

[39] Zhang HF, Sun BS, Qi GS. Comparisons between compressed air-agitated coagulation and mechanically-agitated coagulation. Ind Water Wastewater, 2004, 35(5): 51-53. [张海丰, 孙宝盛, 齐庚申. 压缩空气摚拌与机械摚拌混凝的比较. 工业用水与废水, 2004, 35(5): 51-53.] 